\title{
A Matrix Scheme Enabling Dynamic Bandwidth Allocation in Metro- Access Optical Network based on OFDM-PON
}

\author{
Weilun Xie, Chaoqin Gan, Qi Shao, Wei Li, Yiqin Fang \\ Key Laboratory of Specialty Fiber Optics and Optical Access Networks, School of \\ Communication and Information Engineering, Shanghai University, \\ No. 149, Yanchang Road, Zhabei District, 200072 Shanghai, China \\ *Chaoqin Gan: cqgan@shu.edu.cn
}

Keywords: OFDM-PON; Dynamic bandwidth allocation (DBA); multi-granularity; access network; matrix.

\begin{abstract}
In this paper, an algorithm of dynamic bandwidth allocation (DBA) is proposed to achieve multi-granularity dynamic bandwidth allocation in multi-wavelength in Metro-Access Optical Network based on OFDM-PON. The DBA mainly focuses on the inner ONU bandwidth allocation. An inner ONU queue is proposed based on OFDM-PON. By the DBA, bandwidth of different granularities can be assigned efficiently.
\end{abstract}

\section{Introduction}

Passive optical networks (PONs) have emerged as a highly attractive broadband access solution to meet the increasing demand of bandwidths from various services [1]. At present, the PON is mainly based on TDM such as EPON and GPON. However, the bandwidth provided for each end-user is limited. In the WDM-PON, Although each user can be assigned a dedicated wavelength to enjoy the full bandwidth [2], It can't meet the QoS demand and the information rate of different services. In contrast, the OFDM-PON is highly flexible in terms of supporting multiple granularities of bandwidth through advanced digital signal processing (DSP) techniques. It also has high bandwidth efficiency through high efficient digital modulation and dynamic resource management [3].Thus, more and more discussions about the protocols and algorithms for OFDM-PON come up. In this paper, an algorithm of dynamic bandwidth allocation (DBA) and an inner ONU queue are proposed in multi- wavelength optical access network (OAN) based on OFDM-PON. A mathematic model based on matrix theory is also proposed for a typical PON architecture. The DBA can assign not only the wavelength and the number of subcarriers but also the timeslot and the modulation format of subcarriers.

\section{Network architecture}

The next-generational OAN will be a hybrid PON containing a large number of ONUs [4]. And ONUs can be

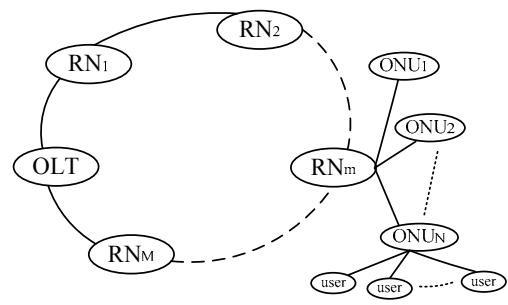

Fig. 1. Network Architecture

divided into many groups on the basis of region or logical relation. In this paper, we focus on the OFDM-PON scheme in the OAN. The network architecture is based on a fiber ring. Many "trees" are interconnected with the ring by the RN. This network consists of one OLT, M RNs and N ONUs in each RN. Each ONU connects with X users (see Fig.1). In this architecture, K wavelengths are provided by the OLT, each RN commonly drops $\mathrm{N}$ wavelengths, and one wavelength serves for one 
ONU. Among the ONUs, some employ the OFDM-PON scheme, the other employs EPON and WDM-PON. To explain clearly, REPORT and GATE messages are introduced here [5]. But, the GATE frame is upgraded to support additional bytes to carry messages of wavelengths and subcarriers to achieve dynamic bandwidth allocation in OFDM-PON.

\section{Mathematical model}

There are K wavelengths in the OAN. As some wavelengths will be used to support the expansion of the OAN, there should be $\mathrm{K} \geqq \mathrm{MN}$. Here, to indicate whether a wavelength is free or not, matrix $\Lambda$ is introduced as follow:

$$
\Lambda=\left[\begin{array}{cccc}
\lambda_{11} & \lambda_{12} & \ldots & \lambda_{1 K} \\
\lambda_{21} & \lambda_{22} & \ldots & \lambda_{2 K} \\
\ldots \ldots & \ldots & \ldots & \ldots \\
\lambda_{M N 1} & \lambda_{M N 2} & \ldots & \lambda_{M N K}
\end{array}\right]_{M N \times K}
$$

$\lambda_{m n}=1 / 0$ indicates the $\mathrm{k}$-th wavelength is/isn't employed by the (mn)-th ONU. Here, it is assumed that the OFDM-PON carries L+2 subcarriers in total. Among them, two subcarriers are used to convey the control message between the OLT and the ONU. After the OLT receives bandwidth requirements from all ONUs, it makes the decision through the DBA. After that, it sends the message of bandwidth to the ONU respectively. Here, matrix P is defined to indicate the employment of each subcarrier:

$$
P=\left[\begin{array}{cccc}
p_{11} & p_{12} & \ldots & p_{1 L} \\
p_{21} & p_{22} & \ldots & p_{2 L} \\
\ldots & \ldots & \ldots & \ldots \\
p_{X 1} & p_{X 2} & \ldots & p_{X L}
\end{array}\right]_{X \times L}
$$

$P_{x l}=1 / 0$ indicates the 1-th subcarrier is/isn't employed by the $\mathrm{x}$-th user sometime. OFDM subcarriers can be dynamically assigned to different services and/or users by the corresponding bandwidth demand. On the one hand, as the bandwidth of one subcarrier can be further divided into smaller granularity in timeslot level, it can be shared by many users or services. Each user or service can occupy different timeslots. On the other hand, as a subcarrier may be able not to meet a user's demand, more subcarriers may be assigned to the user in such a case.

Moreover, in the OFDM-PON, the spectral efficiency can be increased cost-effectively by employing multi-level signal, such as M-ary Quadrature Amplitude Modulation (QAM). Here, a modulation-format matrix $\mathrm{F}$ is defined to indicate the bandwidth of each subcarrier. All subcarriers are assumed to have I level modulation formats, such as 4QAM, 8QAM, 16QAM. As the symbol rate can be changed adaptively, $\mathrm{RB}$ is used to indicate the rate of symbol of each subcarrier and $h_{i}$ is defined to indicate the quality of information of a symbol with i-th level modulation. Matrix $\mathrm{F}$ is proposed as the following equation:

$$
F=\left[\begin{array}{cccc}
f_{11} & f_{12} & \ldots & f_{1 L} \\
f_{21} & f_{22} & \ldots & f_{2 L} \\
\ldots & \ldots & \ldots & \ldots \\
f_{I 1} & f_{I 2} & \ldots & f_{I L}
\end{array}\right]_{I \times L}
$$

$f_{i l}$ indicates the bandwidth of the 1-th subcarrier with the i-th level modulation. It can be calculated through the equation as follow:

$$
f_{i l}=R_{B} \times h_{i l}
$$

In the OAN, each user is assumed to possess $\mathrm{Y}$ services at most. To know the bandwidth of each service clearly, matrix $\mathrm{E}$ is defined as the following equation:

$$
E=\left[\begin{array}{cccc}
E_{11} & E_{12} & \ldots & E_{1 Y} \\
E_{21} & E_{22} & \ldots & E_{2 Y} \\
\ldots & \ldots & \ldots & \ldots \\
E_{X 1} & E_{X 2} & \ldots & E_{X Y}
\end{array}\right]_{X \times Y}
$$


$E_{x y}$ indicates the bandwidth of the y-th service owned by $u \operatorname{ser}_{x}$. Matrix $D_{x}$ is proposed to indicate the services of a user:

$$
D_{x}=\left[\begin{array}{cccc}
d_{11} & 0 & \ldots & 0 \\
0 & d_{22} & \ldots & 0 \\
\ldots & \ldots & \ldots & \ldots \\
0 & 0 & \ldots & d_{Y Y}
\end{array}\right]_{Y \times Y}
$$

$d_{y y}=1 / 0$ indicates the $\mathrm{y}$-th service is/isn't possessed by user $_{x}$. Matrix $B_{\text {require }}$ is proposed to indicate the bandwidth demand of each user. It can be seen in the equation as follow:

$$
B_{\text {require }}=\left[\begin{array}{c}
B_{\text {require-1 }} \\
B_{\text {require-2 }} \\
\cdots \\
B_{\text {require- } X}
\end{array}\right]_{X \times 1}
$$

The bandwidth required by each user can be calculated through the above matrices. The bandwidth demand of $\operatorname{user}_{x}\left(B_{\text {require }}\right)$ is calculated as follow:

$$
B_{\text {require }-x}=E_{x \bullet} \times D_{x}
$$

In consideration of the Service-Level Agreement (SLA), let matrix $B_{\min }$ indicate the minimum bandwidth guaranteed of all users as follow:

$$
B_{\min }=\left[\begin{array}{c}
b_{\min -1} \\
b_{\min -2} \\
\cdots \\
b_{\min -X}
\end{array}\right]_{X \times 1}
$$

$b_{\min -x}$ indicates the minimum bandwidth guaranteed of $\operatorname{user}_{x}\left(b_{\min -x}\right.$ is different from $b_{\min }$ [6]).

In the OAN, there are various kinds of services. Among them, some are more important than others and their bandwidth requirements must be guaranteed. The minimum bandwidth guaranteed of user $_{x}$ is calculated as the following equation:

$$
b_{\min -x}=\sum_{y} E_{x y}
$$

In (10), $E_{x y}$ indicates the bandwidth of the service must be guaranteed.

\section{Bandwidth allocation}

In Fig.2. The queuing discipline is as follows: packets from each user are classified into queues according to their priority by the classifier. If "guaranteed" buffer belonged to the high-priority service is full, the corresponding packets can occupy the place of lower-priority packets. Alternatively, if "others" buffer belonged to the low-priority service is full, the corresponding packets will be dropped. Customarily, after the polling, the packets in "guaranteed" queue will be transmitted prior to the packets in "others" queues.

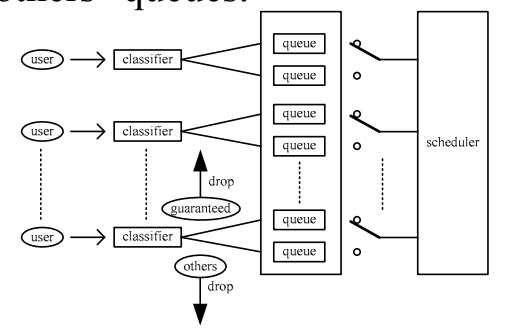

Fig. 2. Schedule of inner ONU

The character of multi-granularity bandwidth makes OFDM-PON be superior to other PONs. It has two modes of bandwidth schedules: In the first mode, demands of users are met by changing the 
number of subcarriers. In the second mode, demands of users are met by changing modulation formats of subcarriers.

In the bandwidth allocation, the OLT allocates some subcarriers among $L$ subcarriers to all users for $b_{\min -x}$ at first. The number and the modulation format of subcarriers to be assigned for $b_{\min -x}$ can be calculated as follow:

If $(a-1) f_{I l}<b_{\min -x} \leq a f_{I l}$ ( $a$ is a integer), the OLT assigns $a$ subcarriers to $u \operatorname{ser}_{x}$ for $b_{\min -x}$. Then, if $(a-1) f_{I l}+f_{(i-1) l}<b_{\min -x} \leq(a-1) f_{I l}+f_{i l}$, let $b_{\min -x}^{\text {assign }}=f_{1 x}\left(b_{\min -x}^{\text {assign }}\right.$ is the bandwidth of subcarriers assigned to meet $b_{\min -x}$ ).

When the bandwidth requirements in the AN change, the OLT waits until bandwidth requirements from all ONUs are received in the cycle of polling. Then it operates the DBA to determine bandwidths for users:

If $B_{\text {require- } x}<b_{\min -x}$, then $B_{\text {require }-x}^{\text {assign }}=b_{\min -x}$. If $B_{\min -x}^{\text {assign }}>B_{\text {require }-x}>b_{\min -x}$, then $B_{\text {require }-x}^{\text {assign }}=b_{\min -x}^{\text {assign }}$.

If $B_{\text {require- } x}>b_{\min -x}^{\text {assign }}$, the OLT upgrades the modulation level of subcarriers having been assigned to guarantee $b_{\min -x}$ until $B_{\text {require }-x}<b_{\min -x}^{a s s i g n}$. And then $B_{\text {require }-x}^{\text {assign }}=B_{\text {require }-x}$.

If "subcarriers of $b_{\min -x}^{\text {assign }}$ are at the highest modulation level" and $B_{\text {require }-x}>b_{\min -x}^{\text {assign }}$, the OLT computes whether there are subcarriers available through the following equations:

$$
\begin{aligned}
{\left[\begin{array}{llll}
p_{o-1} & p_{o-2} & \ldots & p_{o-L}
\end{array}\right]_{1 \times L} } & =\left[\begin{array}{llll}
1 & 1 & \ldots & 1
\end{array}\right]_{1 \times X} \times P=P_{o} \\
p_{o-l} & =\sum_{x=1}^{X} p_{x l}
\end{aligned}
$$

When $p_{o-l}=0$, it indicates the $l$-th subcarrier is available. Then the OLT assigns the subcarrier to $u_{\text {ser }}$. And the procedure above is repeated until $B_{\text {require }-x}^{\text {assign }}=B_{\text {require }-x}$ ( $B_{\text {require }-x}^{\text {assign }}$ is the bandwidth assigned to users to meet their bandwidth requirements).

If $p_{o-l} \neq 0$, it indicates that there is no subcarrier available in the OFDM-PON. Then the OLT computes the excessive bandwidths from all lightly-loaded subcarriers and assigns them to users not having enough bandwidth. Matrix $f_{\text {excess }}$ indicates the excess bandwidth of all subcarriers:

$$
f_{\text {excess }}=\left[\begin{array}{c}
f_{\text {excess }-1} \\
f_{\text {excess }-2} \\
\ldots \\
f_{\text {excess }-L}
\end{array}\right]_{L \times I}
$$

$f_{\text {excess }-l}$ is the excess bandwidth of the $l$-th subcarrier and can be calculated through the following equation:

$$
f_{\text {excess-l }}=f_{\text {il }}-f_{\text {il }}^{\text {occupy }}
$$

$f_{i l}^{\text {occupy }}$ is the portion of $f_{i l}$ assigned to users. The total excess bandwidth $\left(B_{\text {excess }}^{\text {total }}\right)$ can be calculated as follow:

$$
B_{\text {excess }}^{\text {total }}=\left[\begin{array}{llll}
1 & 1 & \ldots & 1
\end{array}\right]_{1 \times L} \times f_{\text {excess }}
$$

Let $T_{\text {cycle }}$ be the granting cycle. During a $T_{\text {cycle }}$, all ONUs can transmit their data and REPORTs to the OLT. When ONUs transmit bandwidth requirement to the OLT, users transmit message to ONUs at the same time. The ONU keeps these messages in its buffer until next time to transmit bandwidth requirement. The excess bandwidth updates with the change of users' requirements:

$$
B_{\text {excess }}^{\text {total }}=\operatorname{update}\left(B_{\text {excess }}^{\text {total }}\right)
$$

To guarantee the fairness among users, a fair-excess-allocation scheme is adopted to assign the 
portions to users according to their bandwidth demands [7]. Let $B_{\text {require }-x}^{\text {excess }}$ be the bandwidth requested by user $_{x}$ and $B_{\text {total }}^{\text {excess }}$ be the total bandwidth required by all users. They can be calculated as follows:

$$
\begin{aligned}
& B_{\text {require- } x}^{\text {excess }}=B_{\text {require- } x}-B_{\text {require- } x}^{\text {assign }} \\
& B_{\text {total }}^{\text {excess }}=\sum_{x=1}^{X} B_{\text {require }-x}^{\text {exces }}
\end{aligned}
$$

After the OLT receives bandwidth requirements, it assigns the excess bandwidth ( $B_{\text {excess }}^{a \text { assign }}$ ) to users. To avoid waste of bandwidth, a scheme is adopted here :

$$
B_{\text {excess }-x}^{\text {assign }}=\left\{\begin{array}{cl}
\frac{B_{\text {require- } x}^{\text {excess }} \times B_{\text {excess }}^{\text {total }}}{B_{\text {total }}^{\text {excess }}}, & \left(B_{\text {require- } x}^{\text {excess }}>\frac{B_{\text {require-x }}^{\text {excess }} \times B_{\text {excess }}^{\text {total }}}{B_{\text {total }}^{\text {excess }}}\right) \\
B_{\text {require- } x}^{\text {excess }}, & \left(B_{\text {require- } x}^{\text {excess }}<\frac{B_{\text {require }-x}^{\text {excess }} \times B_{\text {excess }}^{\text {total }}}{B_{\text {total }}^{\text {excess }}}\right)
\end{array}\right.
$$

$W$ is defined to indicate the utilization ratio of bandwidth, and it can be calculated through the following equation:

$$
W=\frac{B_{\text {require }}^{\text {total }}}{\sum_{l=1}^{L} f_{i l}}=\frac{\sum_{x=1}^{X}\left(E_{x \bullet} \times D_{x}\right)}{R_{B} \times P_{0} \times H}
$$

If $W>1$, it means the bandwidth to meet the demand of all users.

If $W<1$, the OLT checks whether there are wavelengths available by the following method:

$$
\left[\begin{array}{llll}
1 & 1 & \ldots & 1
\end{array}\right]_{1 \times M N} \times \Lambda=\left[\begin{array}{llll}
\lambda_{1} & \lambda_{2} & \ldots & \lambda_{k}
\end{array}\right]_{1 \times K}
$$

If $\lambda_{k}=0$, it indicates the $k$-th wavelength is available. Then the OLT schedules the free wavelength to the ONU. And the bandwidth of this wavelength is scheduled through the DBA introduced above.

According to the DBA introduced above, the following formulations must be satisfied to avoid message loss:

$$
\begin{gathered}
\int_{t_{o}}^{t} B_{\text {require }}^{\text {total }} d t-\operatorname{Buffer}\left(t_{o}\right) \leq \int_{t o}^{t} \sum_{l=1}^{L} f_{i l} d t \\
\int_{t_{o}}^{t} \sum_{x=1}^{X}\left(E_{x} \times D_{x}\right) d t-\operatorname{Buffer}\left(t_{o}\right) \leq \int_{t_{o}}^{t}\left(R_{B} \times P_{0} \times H\right) d t
\end{gathered}
$$

Here, Buffer $\left(t_{o}\right)$ is the available space of the buffer of ONU. On the contrary, if

$$
\int_{t_{o}}^{t} \sum_{x=1}^{X}\left(E_{x \bullet} \times D_{x}\right) d t-\operatorname{Buffer}\left(t_{o}\right)>\int_{t_{o}}^{t}\left(R_{B} \times P_{0} \times H\right) d t
$$

That means the buffer is full and it cannot accept any packet. Thus, the packet arrived later will be dropped.

\section{Summary}

A DBA based on matrix theory is proposed in this paper. It can achieve multi-granularity bandwidth allocation in the OAN based on OFDM-PON, such as the schedule of both the number and the modulation format of subcarriers. Users can share the bandwidth of a subcarrier through TDM mode. In the paper, some basic rules are also proposed to prevent the message packet from dropping. The above discussion shows the DBA can efficiently cope with various bandwidth requirements in the OAN. 


\section{Acknowledgment}

This work is supported by Programs of Natural Science Foundation of China (No.61132004, 61275073 and 61420106011), Shanghai Science and Technology Development Funds (No. 13JC1402600 and 14511100100), Shanghai Leading Academic Discipline Project (No.S30108), Shanghai Economics and Information Project (No.CXY-2013-93) and Key Laboratory of Specialty Fiber Optics and Optical Access Networks, Shanghai University (No.SKLSFO2012-05).

\section{References}

[1] Forzati Marco, Gavler Anders. "Flexible next-generation optical access. Transparent Optical Networks (ICTON)," 2013 15th International Conference, Cartagena, Spain, 2013:1-8.

[2] X.F. Cheng, Y.K. Yeo, C. Li, X.W. Xu. "Wavelength-agile Optical Access Networking System," Communications and Photonics Conference and Exhibition (ACP), Asia, 2011: 1-7.

[3] Wei Wei, Ting Wang, Dayou Qian, Junqiang Hu. "MAC Protocols for Optical Orthogonal Frequency Division Multiple Access (OFDMA)-based Passive Optical Networks," Optical Fiber communication/National Fiber Optic Engineers Conference (OFC/NFOEC), San Diego, CA, 2008: 1 - 3 .

[4] X Jiang. "Progress and Challenges for High Speed Optical Access Networks," Wireless and Optical Communications Conference (WOCC), 2011: 1 - 3.

[5] M. P. McGarry, M. Reisslein. "WDM Ethernet Passive Optical Networks," Communications Magazine, IEEE, 2006; 44 (2): 15 - 22.

[6] C. M. Assi et al. "Dynamic bandwidth allocation for Quality-of-Service over Ethernet PONs," IEEE JSAC, 2003; 2 1: 1467-1477.

[7] G.Kramer, B.Mukherjee, G.Pesavento. "IPACT: A Dynamic Protocol for an Ethernet PON (EPON)," Communications Magazine, IEEE, 2002; 40: 74 - 80. 